Pacific Journal of Mathematic 


\title{
THE GARABEDIAN FUNCTION OF AN ARBITRARY COMPACT SET
}

\author{
ERIC P. SMITH
}

\begin{abstract}
If the outer boundary of the compact plane set $E$ is the union of finitely many disjoint analytic Jordan curves, the Garabedian function of $E$ is a familiar object. J. Garnett and S. Y. Havinson have each asked whether the Garabedian functions of a decreasing sequence of such sets must converge. The present paper shows that they do converge. This fact leads to a natural definition of the Garabedian function of an arbitrary compact plane set. As an intermediate step, an approximate formula is obtained for the analytic capacity of the union of a compact set $E$ and a small disc not intersecting $E$.
\end{abstract}

1. Prerequisites and notation. Good introductions to Analytic Capacity are given in [2], pp. 1-26, and [1], Ch. 8; and so we shall give only a brief outline.

$C$ denotes the complex plane. $S^{2}$ denotes the extended complex plane with its usual topology. $D(z ; r)$ denotes the closed disc with centre $z$ and radius $r$.

Let $E$ be a compact subset of $C . \Omega(E)$ denotes the component of $S^{2} \backslash E$ containing $\infty$. The outer boundary of $E$ is the boundary $\partial \Omega(E)$ of $\Omega(E)$. The analytic capacity of $E$ is:

$$
\gamma(E)=\sup \left\{\left|g^{\prime}(\infty)\right|: g \text { analytic on } \Omega(E),|g|<1\right\} \text {. }
$$

This supremum is attained by a unique function, the Ahlfors function of $E$ ([1], p. 197).

$\mathscr{S}$ will denote the class of all compact plane sets whose outer boundary is the union of finitely many pairwise disjoint analytic Jordan curves. Let $E \in \mathscr{S}$, and write $\Omega=\Omega(E)$. The Hardy space $H^{p}(\Omega)$ $(0<p<\infty)$ is the space of all analytic functions $g$ on $\Omega$ such that there exists a harmonic function $u$ on $\Omega$ with $|g|^{p}<u$. If $g \in H^{p}(\Omega)$ then $g$ has a finite nontangential limit $g(z)$ at almost every point $z \in \partial \Omega . \quad H^{2}(\Omega)$ is a separable Hilbert space with the inner product:

$$
(g, h)=\int_{\hat{\partial} \Omega} g(z) h(z)^{*} d s \quad\left(g, h \in H^{2}(\Omega)\right) .
$$

If $\zeta \in \Omega$ there is a unique function $K(z, \zeta)$ in $H^{2}(\Omega)$, the Szegö kernel function, such that:

$$
g(\zeta)=\int_{\partial \Omega} g(z) K(z, \zeta)^{*} d s \quad\left(g \in H^{2}(\Omega)\right) .
$$

$K(z, \zeta)$ is the inner product between the functionals on $H^{2}(\Omega)$ given 
by evaluation at $z$ and $\zeta$, so that $K(z, \zeta)=\sum u_{n}(z) u_{n}(\zeta)^{*}$, whenever $\left\{u_{n}\right\}$ is an orthonormal basis for $H^{2}(\Omega)$. The Garabedian function is most easily defined for our purpose as:

$$
\psi(z)=\frac{2 \pi}{i} \gamma(E)^{2} K(z, \infty)^{2} .
$$

See [2], pp. 13-23.

Throughout, $E$ will be a compact plane set, $\Omega=\Omega(E)$, and $f$ will be the Ahlfors function of $E$. If $E \in \mathscr{S}, K(z, \zeta)$ will denote its Szegö kernel function, and $\psi$ its Garabedian function.

We shall use the following results.

1.1. Let $\left\{E_{n}\right\}$ be a decreasing sequence of compact sets with intersection $E$. Let $f_{n}$ be the Ahlfors function of $E_{n}$. Then $f_{n} \rightarrow f$ uniformly on compact subsets of $\Omega$, and $\gamma\left(E_{n}\right) \rightarrow \gamma(E)$ ([1], p. 198).

1.2. Let $E \in \mathscr{S}$. Then:

(1) $f$ and $\psi$ are analytic across $\partial \Omega$.

(2) $|f|=1$ on $\partial \Omega$.

(3) $f(z) \psi(z) d z \geqq 0$ on $\partial \Omega$.

(4) $\psi(\infty)=1 /(2 \pi i)$.

(5) $K(\infty, \infty)=1 /(2 \pi \gamma(E))$.

([2], pp. 18-23).

1.3. Let $E, F$ be compact, $\gamma(E)=0$. Then $\gamma(E \cup F)=\gamma(F)$ (an immediate consequence of [2], Theorem 1.4, pp. 10-11).

1.4. Let $E$ be compact, $0 \notin E, E \subset D(0 ; R)$. Denote by $E_{*}$ the inversion of $E$ in the unit circle. Then:

$$
\gamma\left(E_{*}\right) \geqq \gamma(E) / 8 R^{2}
$$

(proof similar to [1], Lemma 12.2, p. 229).

Finally we need the following result on Hilbert spaces.

Proposition 1.5. Let $h$ be a separable Hilbert space, and let $\left\{u_{n}\right\}$ be a sequence of vectors in $h$ whose closed linear span is $h$. Suppose that the infinite matrix $T$ given by $T_{i j}=\left(u_{j}, u_{i}\right)$ is bounded and invertible (as an operator on $l_{2}$ ). Then for every bounded linear functional $f$ on $h$ the sequence $\left\{f\left(u_{i}\right)\right\}$ is square-summable and:

$$
\|f\|^{2}=\sum_{i, j=1}^{\infty}\left(T^{-1}\right)_{i j} f\left(u_{i}\right) f\left(u_{j}\right)^{*} .
$$

Proof. $T$ is positive, and so is the matrix of a positive operator 
$P \in B\left(l_{2}\right) . P$ has a positive square root $P^{1 / 2}$, which is invertible since $P$ is invertible. For $i=1,2,3, \cdots$, write $w_{i}=P^{1 / 2} e_{i}$, where $e_{i}$ is the vector with 1 in its $i$ th place and 0 elsewhere. Since $P^{1 / 2}$ is invertible, $l_{2}$ is the closed linear span of the $w_{i} .\left(w_{j}, w_{i}\right)=\left(P^{1 / 2} e_{j}, P^{1 / 2} e_{i}\right)=\left(P e_{j}, e_{\imath}\right)=$ $T_{i j}=\left(u_{j}, u_{i}\right)$; so we can define a unitary $J: l_{2} \rightarrow h$ by $J\left(w_{i}\right)=u_{i}$ for all $i$, extended to the whole of $l_{2}$ by linearity and continuity. The bounded linear functional $J^{*} f$ on $l_{2}$ is represented by some $s \in l_{2}$. $\left(e_{i}, P^{1 / 2} s\right)=\left(P^{1 / 2} e_{i}, s\right)=\left(w_{i}, s\right)=\left(J^{*} f\right)\left(w_{i}\right)=f\left(J w_{i}\right)=f\left(u_{i}\right)$. Hence $\left\{f\left(u_{i}\right)\right\}$ is square-summable. Also:

$$
\begin{aligned}
f \|^{2} & =\|s\|^{2}=\left(P^{-1}\left(P^{1 / 2} s\right),\left(P^{1 / 2} s\right)\right) \\
& =\sum_{i, j=1}^{\infty}\left(T^{-1}\right)_{i j}\left(e_{i}, P^{1 / 2} s\right)\left(e_{j}, P^{1 / 2} s\right)^{*}=\sum_{i, j=1}^{\infty}\left(T^{-1}\right)_{i j} f\left(u_{i}\right) f\left(u_{j}\right)^{*} .
\end{aligned}
$$

2. The slope function. The purpose of this section is to establish Theorem 2.2, which gives an expression, up to first order in $\varepsilon$, for the analytic capacity of a set of the form $E \cup D(z ; \varepsilon)$, where $E \in \mathscr{S}$ and $z \in \Omega(E)$. This will be extended to arbitrary compact sets $E$ in $\S 3$. First we need a lemma which gives bounds on the Szegö kernel function.

Lemma 2.1. Let $E \in \mathscr{S}, \zeta \in \Omega(E), \zeta \neq \infty$. Let $r, R$ be the least and greatest distances of points of $E$ from $\zeta$. Then:

$$
\frac{r^{2}}{16 \pi R^{2} \gamma(E)} \leqq K(\zeta, \zeta) \leqq \frac{8 R^{2}}{2 \pi r^{2} \gamma(E)} .
$$

Proof. We prove the upper bound: the lower one is similar. We may assume that $\zeta=0$. Let $g \in H^{2}(\Omega),\|g\| \leqq 1$. Denote inversion in the unit circle by $*$. Define $g_{*}$ on $\Omega_{*}$ by $g_{*}(z)=g\left(z_{*}\right)^{*}$. Clearly $g_{*} \in H^{2}\left(\Omega_{*}\right)$ and $\left\|g_{*}\right\| \leqq 1 / r$. Hence:

$$
|g(0)|^{2}=\left|g_{*}(\infty)\right|^{2} \leqq \frac{\left\|g_{*}\right\|^{2}}{2 \pi \gamma\left(E_{*}\right)} \leqq \frac{1}{2 \pi r^{2} \gamma\left(E_{*}\right)} \leqq \frac{8 R^{2}}{2 \pi r^{2} \gamma(E)}
$$

by 1.4. So:

$$
K(0,0)=\sup \left\{|g(0)|^{2}: g \in H^{2}(\Omega),\|g\| \leqq 1\right\} \leqq \frac{8 R^{2}}{2 \pi r^{2} \gamma(E)} .
$$

There is a simpler bound for the Garabedian function: for, in the above notation:

$$
\left|\psi(\zeta)-\frac{1}{2 \pi i}\right|=\left|\frac{1}{2 \pi i} \int_{\partial \Omega} \frac{\psi(z) d z}{z-\zeta}\right| \leqq \frac{1}{2 \pi r} \int_{\partial \Omega}|\psi| d s=\frac{\gamma(E)}{2 \pi r} .
$$

THEOREM 2.2. Let $E \in \mathscr{S}$. There is a positive real-valued func- 
tion $a_{E}(\zeta)$, the slope function of $E$, defined on $\Omega$, with the property that for all $\zeta \in \Omega$ :

$$
\gamma(E \cup D(\zeta ; \varepsilon))=\gamma(E)+\varepsilon a_{E}(\zeta)+O\left(\varepsilon^{2}\right) .
$$

$a_{E}(\zeta)$ is given explicitly by:

$$
a_{E}(\zeta)=2 \pi|\psi(\zeta)|\left\{1-|f(\zeta)|^{2}\right\}
$$

The bound in the error term depends only on $\gamma(E)$ and on the ratio of the greatest and least distances of points of $E$ from $\zeta$.

Proof. We may suppose that $\zeta=0$. Let $r, R$ be the least and greatest distances of points of $E$ from 0 . Note that $E \subset D(0 ; R)$, so that $\gamma(E) \leqq R$. We shall prove the theorem by showing that:

$$
\begin{aligned}
\varepsilon & <10^{-5}(r / R)^{5} \gamma(E) \Longrightarrow & \left|\gamma(E \cup D(0 ; \varepsilon))-\gamma(E)-\varepsilon a_{E}(0)\right| \\
& \leqq 10^{9}(R / r)^{10} \gamma(E)^{-1} \varepsilon^{2} . &
\end{aligned}
$$

Fix $\varepsilon<10^{-5}(r / R)^{5} \gamma(E)$. Since $r<R$ and $\gamma(E) \leqq R$, we have $\varepsilon<$ $10^{-5} r$; so $D(0 ; \varepsilon)$ does not meet $E$. Write $E_{1}=E \cup D(0 ; \varepsilon), \Omega_{1}=\Omega\left(E_{1}\right)$, $H^{2}=H^{2}(\Omega), H_{1}^{2}=H^{2}\left(\Omega_{1}\right), \gamma=\gamma(E), \gamma_{1}=\gamma\left(E_{1}\right)$. Choose an orthonormal basis $\left\{u_{n}\right\}$ for $H^{2}$. Now we can use the Cauchy integral to express any element of $H_{1}^{2}$ as the sum of an element of $H^{2}$ and an element of $H^{2}\left(S^{2} \backslash D(0 ; \varepsilon)\right)$. The latter space is the closed linear span of $\left\{z^{-n}: n \geqq 0\right\}$. It follows that if, for $n \geqq 1, v_{n}$ is any function analytic on $\bar{\Omega}$ except for a pole of order $n$ at 0 , then $H_{1}^{2}$ is the closed linear span of $\left\{u_{n}\right\} \cup$ $\left\{v_{n}\right\}$. To be specific, we shall put:

$$
v_{n}(z)=\frac{\varepsilon^{n-1 / 2}}{\sqrt{(2 \pi)} K(0,0)} \frac{K(z, 0)}{z^{n}} .
$$

1.2 (5) says that $1 /\left(2 \pi \gamma_{1}\right)$ is the square of the norm of evaluation at $\infty$ in $H_{1}^{2}$. Our proof consists of calculating this by applying Proposition 1.5 to $\left\{u_{n}\right\} \cup\left\{v_{n}\right\}$.

We shall calculate various bounds now, so as not to break continuity later. Throughout, "\| ||" and "norm" will refer to the norm of an element of a Hilbert space, or the norm of an infinite matrix considered as a bounded operator on $l_{2}$; and " \|\|$_{\infty}$ " will denote the supremum of the absolute value of a function on the set $D(0 ; \varepsilon)$.

Let $z_{0} \in C,\left|z_{0}\right| \leqq \varepsilon$. For $n \geqq 1$ :

$$
u_{n}\left(z_{0}\right)=\frac{1}{2 \pi i} \int_{|z|=r / 2} \frac{u_{n}(z) d z}{z-z_{0}}
$$

Hence: 


$$
\left\|u_{n}\right\|_{\infty} \leqq \frac{1}{2 \pi(r / 2-\varepsilon)} \int_{|z|=r / 2}\left|u_{n}\right| d s,
$$

so that by Schwarz's inequality:

$$
\left\|u_{n}\right\|_{\infty}^{2} \leqq \frac{\pi r}{4 \pi^{2}(r / 2-\varepsilon)^{2}} \int_{|z|=r / 2}\left|u_{n}\right|^{2} d s
$$

Summing over $n$ and using Lemma 2.1 gives:

$$
\sum\left\|u_{n}\right\|_{\infty}^{2} \leqq \frac{r}{4 \pi(r / 2-\varepsilon)^{2}} \int_{|z|=r / 2} K(z, z) d s
$$

$$
\leqq \frac{r \cdot \pi r}{4 \pi(r / 2-\varepsilon)^{2}} \frac{8(R+r / 2)^{2}}{2 \pi(r / 2)^{2} \gamma} \leqq 3 R^{2} r^{-2} \gamma^{-1}
$$

since $\varepsilon<10^{-5} r$ and $r<R$. Analogous computation gives:

$$
\sum\left\|u_{n}^{\prime}\right\|_{\infty}^{2} \leqq 50 R^{2} r^{-4} \gamma^{-1} \text {. }
$$

In particular:

$$
\sum\left|u_{n}^{\prime}(0)\right|^{2} \leqq 50 R^{2} r^{-4} \gamma^{-1}
$$

Next we want a bound for $\left\|d^{k} / d z^{k} K(z, 0)\right\|_{\infty}$. Let $z_{0} \in C,\left|z_{0}\right| \leqq \varepsilon$. Then for $k \geqq 1$ and for all $s<r$ :

$$
\begin{aligned}
\left|\frac{d^{k}}{d z^{k}} K(z, 0)\right|_{z=z_{0}} \mid & =\left|\frac{k !}{2 \pi i} \int_{|z|=s} \frac{K(z, 0) d z}{\left(z-z_{0}\right)^{k+1}}\right| \\
& \leqq \frac{k !}{2 \pi} \frac{2 \pi s}{(s-\varepsilon)^{k+1}} \frac{8 R(R+s)}{2 \pi r(r-s) \gamma} .
\end{aligned}
$$

(Here we have estimated $|K(z, 0)|$ by $K(z, z)^{1 / 2} K(0,0)^{1 / 2}$ and then used Lemma 2.1.) In particular, putting $s=k r /(k+1)$ :

$$
\begin{aligned}
\left|\frac{d^{k}}{d z^{k}} K(z, 0)\right|_{z=z_{0}} \mid & \leqq \frac{k !}{2 \pi} \frac{8 R \cdot 2 R}{r(r-(k+1) \varepsilon / k)^{k+1} \gamma} \frac{(k+1)^{k+1}}{k^{k}} \\
& \leqq \frac{(k+1) !}{2 \pi} \frac{16 R^{2}}{r(r-2 \varepsilon)^{k+1} \gamma} e \leqq \frac{7 R^{2}(k+1) !}{r(r-2 \varepsilon)^{k+1} \gamma}
\end{aligned}
$$

This holds also for $k=0$ by Lemma 2.1. Hence for $k=0,1,2, \cdots$ :

$$
\left\|\frac{d^{k}}{d z^{k}} K(z, 0)\right\|_{\infty} \leqq \frac{7 R^{2}(k+1) !}{r(r-2 \varepsilon)^{k+1} \gamma} .
$$

We need one more estimate. Since $\varepsilon<10^{-5} r$, (4) gives:

$$
\left\|\frac{d K(z, 0)}{d z}\right\|_{\infty} \leqq \frac{15 R^{2}}{r^{3} \gamma} .
$$

Hence, using Lemma 2.1 and the fact that $\varepsilon<10^{-5} R^{-4} r^{5}$, we have: 


$$
\|K(z, 0)\|_{\infty} \leqq K(0,0)+\varepsilon\left\|\frac{d K(z, 0)}{d z}\right\|_{\infty} \leqq 1.01 K(0,0) \text {. }
$$

We shall imagine the basis $\left\{u_{n}\right\} \cup\left\{v_{n}\right\}$ to be partitioned into three sections. The first section consists of all the $u_{n}$, the second section consists of $v_{1}$ alone, and the third section consists of $v_{2}, v_{3}, v_{4}, \cdots$. The corresponding matrix $T$ of inner products will be in block form:

$$
T=I+M, \quad M=\left[\begin{array}{lll}
A & B^{H} & C^{H} \\
B & D & E^{H} \\
C & E & F
\end{array}\right]
$$

Next we calculate the inner products. Denote inner products in $H_{1}^{2}$ by $($, ). By a statement of the form " $X=Y$ with error $Z$ " we shall mean $|X-Y| \leqq Z$, or $\|X-Y\| \leqq Z$, according to context.

$$
\begin{aligned}
\left(u_{n}, u_{m}\right) & =\int_{\partial \Omega_{1}} u_{n} u_{m}^{*} d s=\int_{\partial \Omega} u_{n} u_{m}^{*} d s+\int_{|z|=\varepsilon} u_{n} u_{m}^{*} d s=\delta_{m n}+2 \pi \varepsilon u_{m}(0)^{*} u_{n}(0) \\
& +\varepsilon \int_{0}^{2 \pi}\left[u_{m}(0)^{*}\left(u_{n}\left(\varepsilon e^{i \theta}\right)-u_{n}(0)\right)+\left(u_{m}\left(\varepsilon e^{i \theta}\right)^{*}-u_{m}(0)^{*}\right) u_{n}\left(\varepsilon e^{i \theta}\right)\right] d \theta \\
\mid\left(u_{n}, u_{m}\right) & -\delta_{m n}-2 \pi \varepsilon u_{m}(0)^{*} u_{n}(0) \mid \leqq 2 \pi \varepsilon^{2}\left(\left\|u_{m}\right\|_{\infty}\left\|u_{n}^{\prime}\right\|_{\infty}+\left\|u_{m}^{\prime}\right\|_{\infty}\left\|u_{n}\right\|_{\infty}\right) .
\end{aligned}
$$

Now the matrix $\left[2 \pi \varepsilon u_{m}(0)^{*} u_{n}(0)\right]$ has norm $2 \pi \varepsilon\left(\sum\left|u_{m}(0)\right|^{2}\left|u_{n}(0)\right|^{2}\right)^{1 / 2}=$ $2 \pi \varepsilon K(0,0) \leqq 8 R^{2} r^{-2} \gamma^{-1} \varepsilon$ by Lemma 2.1. The norm of the matrix $\left[2 \pi \varepsilon^{2}\left(\left\|u_{m}\right\|_{\infty}\left\|u_{n}^{\prime}\right\|_{\infty}+\left\|u_{m}^{\prime}\right\|_{\infty}\left\|u_{n}\right\|_{\infty}\right)\right]$ is at most $4 \pi \varepsilon^{2}\left(\sum\left\|u_{m}\right\|_{\infty}^{2}\left\|u_{n}^{\prime}\right\|_{\infty}^{2}\right)^{1 / 2} \leqq$ $200 R^{2} r^{-3} \gamma^{-1} \varepsilon^{2}$ by (1) and (2). So (see the format (6)):

$$
A=\left[2 \pi \varepsilon u_{m}(0)^{*} u_{n}(0)\right] \text { with error } 200 R^{2} r^{-3} \gamma^{-1} \varepsilon^{2} .
$$

Also, $\|A\| \leqq 8 R^{2} r^{-2} \gamma^{-1} \varepsilon+200 R^{2} r^{-3} \gamma^{-1} \varepsilon^{2} \leqq 9(R / r)^{2} \gamma^{-1} \varepsilon \leqq 9(R / r)^{5} \gamma^{-1} \varepsilon$ since $\varepsilon<10^{-5} r$ and $r<R$. In fact the cruder bound $\|A\| \leqq 2500(R / r)^{5} \gamma^{-1} \varepsilon$ will be sufficient. Observe that, since $\varepsilon<10^{-5}(r / R)^{5} \gamma$, we have also $\|A\| \leqq 1 / 40$.

The $m$ th element of $B$ is:

$$
\begin{aligned}
\left(u_{m}, v_{1}\right)= & \frac{\varepsilon^{1 / 2}}{\sqrt{(2 \pi)} K(0,0)} \int_{\hat{\alpha} \Omega} \frac{K(z, 0)^{*} u_{m} d s}{z^{*}} \\
& +\frac{\varepsilon^{1 / 2}}{\sqrt{(2 \pi)} K(0,0) \Sigma i} \int_{\mid z:=\varepsilon} K(z, 0)^{*} u_{m}(z) d z .
\end{aligned}
$$

Now the second term on the right-hand side is:

$$
\frac{\varepsilon^{-1 / 2}}{\sqrt{(2 \pi)} K(0,0) i} \int_{|z|=\varepsilon}\left(K(z, 0)^{*}-K(0,0)^{*}\right) u_{m}(z) d z
$$

by Cauchy's theorem, and is therefore bounded in magnitude by $\left(2 \pi e^{3 / 2} /(\sqrt{(2 \pi)} K(0,0))\right)\left(15 R^{2} / r^{3} \gamma\right)\left\|u_{m}\right\|_{\infty}$ by $(4)$. So: 


$$
B=\left[\frac{\varepsilon^{1 / 2}}{\sqrt{(2 \pi)} K(0,0)} \int_{\partial \Omega} \frac{K(z, 0)^{*} u_{m} d s}{z^{*}}\right]
$$

$$
\begin{aligned}
& \text { with error } \frac{2 \pi \varepsilon^{3 / 2}}{\sqrt{(2 \pi)} K(0,0)} \frac{15 R^{2}}{r^{3} \gamma}\left(\sum\left\|u_{m}\right\|_{\infty}^{2}\right)^{1 / 2} \\
& \leqq 66 K(0,0)^{-1} R^{3} r^{-4} \gamma^{-3 / 2} \varepsilon^{3 / 2}
\end{aligned}
$$

by (1). The norm of the matrix in the square brackets is at most:

$$
\begin{aligned}
& \frac{\varepsilon^{1 / 2}}{\sqrt{(2 \pi)} K(0,0)}\left(\int_{\partial \Omega} \frac{|K(z, 0)|^{2} d s}{|z|^{2}}\right)^{1 / 2} \\
& \quad \leqq \frac{\varepsilon^{1 / 2}}{\sqrt{(2 \pi)} K(0,0) r}\left(\int_{\partial \Omega}|K(z, 0)|^{2} d s\right)^{1 / 2} \leqq \frac{\varepsilon^{1 / 2}}{\sqrt{(2 \pi)} K(0,0)^{1 / 2} r} .
\end{aligned}
$$

Hence, using Lemma 2.1 and the fact that $\varepsilon<10^{-5}(r / R)^{4} \gamma$, (8) gives $\|B\| \leqq 3 R r^{-2} \gamma^{1 / 2} \varepsilon^{1 / 2}$. The cruder bounds $\|B\| \leqq 1 / 40$ and $\|B\|^{2} \leqq$ $2500(R / r)^{5} \gamma^{-1} \varepsilon$ will suffice. Also, using (8) and the estimates calculated in the last few lines, we have:

$$
\begin{aligned}
B^{H} B= & {\left[\frac{\varepsilon}{2 \pi K(0,0)^{2}} \int_{\partial \Omega} \frac{K(z, 0) u_{m}^{*} d s}{z} \int_{\partial \Omega} \frac{K(z, 0)^{*} u_{n} d s}{z^{*}}\right] } \\
& \text { with error } 20000 R^{6} r^{-8} \varepsilon^{2} .
\end{aligned}
$$

The elements of $C$ are, for $m \geqq 1, n \geqq 2$ :

$$
\begin{aligned}
\left(u_{m}, v_{n}\right)= & \frac{\varepsilon^{n-1 / 2}}{\sqrt{(2 \pi)} K(0,0)} \int_{\partial \Omega} \frac{K(z, 0)^{*} u_{m} d s}{\left(z^{*}\right)^{n}} \\
& +\frac{\varepsilon^{-n+1 / 2}}{\sqrt{(2 \pi)} K(0,0) i} \int_{|z|=\varepsilon} K(z, 0)^{*} u_{m}(z) z^{n-1} d z .
\end{aligned}
$$

Call the first and second terms of the above expression $P_{m n}$ and $Q_{m n}$ respectively. Then:

$$
\begin{aligned}
\|P\| & \leqq \frac{1}{\sqrt{(2 \pi)} K(0,0)}\left(\sum_{n=2}^{\infty} \int_{\partial \Omega} \frac{|K(z, 0)|^{2} d s}{|z|^{2 n}} \varepsilon^{2 n-1}\right)^{1 / 2} \\
& \leqq \frac{1}{\sqrt{(2 \pi)} K(0,0)}\left(\sum_{n=2}^{\infty} \frac{\varepsilon^{2 n-1}}{r^{2 n}} K(0,0)\right)^{1 / 2} \\
& \leqq 3 R r^{-3} \gamma^{1 / 2} \varepsilon^{3 / 2} \leqq(R / r)^{5} \gamma^{-1} \varepsilon .
\end{aligned}
$$

We estimate the integral in the expression for $Q_{m n}$ as follows. Replace $K(z, 0)$ by $K(z, 0)$ minus its Taylor expansion about 0 as far as the term in $z^{n-1}$. By Cauchy's theorem, these added terms do not affect the integral. By Taylor's theorem, $K(z, 0)$ minus its Taylor expansion is bounded on $|z|=\varepsilon$ by $\left(\varepsilon^{n} / n !\right)\left\|d^{n} / d z^{n} K(z, 0)\right\|_{\infty}$, and (4) gives an estimate for that. This procedure gives: 


$$
\begin{aligned}
\|Q\| & \leqq \frac{14 \pi R^{2}}{r \sqrt{(2 \pi)} K(0,0) \gamma}\left(\sum_{n=2}^{\infty} \frac{\varepsilon^{2 n+1}(n+1)^{2}}{(r-2 \varepsilon)^{2 n+2}}\right)^{1 / 2}\left(\sum\left\|u_{m}\right\|_{\infty}^{2}\right)^{1 / 2} \\
& \leqq \frac{14 \pi R^{2}}{r \sqrt{(2 \pi)} \gamma} \frac{16 \pi R^{2} \gamma}{r^{2}} \frac{4 \varepsilon^{5 / 2}}{r^{3}} \frac{\sqrt{3} R}{\sqrt{\gamma} r} \\
& \leqq 6000 R^{5} r^{-7} \gamma^{-1 / 2} \varepsilon^{5 / 2} \leqq(R / r)^{5} \gamma^{-1} \varepsilon .
\end{aligned}
$$

Hence $\|C\| \leqq\|P\|+\|Q\| \leqq 2(R / r)^{5} \gamma^{-1} \varepsilon$. Once again we shall need only $\|C\| \leqq 2500(R / r)^{5} \gamma^{-1} \varepsilon \leqq 1 / 40$.

It is convenient to deal with $\left[\begin{array}{cc}D & E^{H} \\ E & F^{\prime}\end{array}\right]$ as a single matrix. Its $(m, n)$ th element (see (6)) is, for $m \geqq 1, n \geqq 1$ :

$$
\frac{\varepsilon^{m+n-1}}{2 \pi K(0,0)^{2}} \int_{\partial \Omega} \frac{|K(z, 0)|^{2} d s}{\left(z^{*}\right)^{m} z^{n}}+\left(\frac{\varepsilon^{m+n-1}}{2 \pi K(0,0)^{2}} \int_{|z|=\varepsilon} \frac{|K(z, 0)|^{2} d s}{\left(z^{*}\right)^{m} z^{n}}-\delta_{m n}\right) .
$$

Denote by $G_{m n}, H_{m n}$ respectively the first term and the bracketed term of the above expression. We have:

$$
\left|G_{m n}\right| \leqq \frac{\varepsilon^{m+n-1}}{2 \pi K(0,0)^{2}} \frac{1}{r^{m+n}} \int_{\partial \Omega}|K(z, 0)|^{2} d s=\frac{\varepsilon^{m+n-1}}{2 \pi K(0,0) r^{m+n}} .
$$

Hence $\|G\| \leqq(1 /(2 \pi \varepsilon K(0,0))) \sum_{n=1}^{\infty} \varepsilon^{2 n} / r^{2 n} \leqq 9 R^{2} r^{-4} \gamma \varepsilon \leqq 9(R / r)^{5} \gamma^{-1} \varepsilon . \quad H$ is trickier to deal with. We have:

$$
\begin{aligned}
H_{n n} & =\frac{1}{2 \pi K(0,0)^{2} \varepsilon} \int_{|z|=\varepsilon}|K(z, 0)|^{2} d s-1 \\
& =\frac{1}{2 \pi i K(0,0)^{2}} \int_{|z|=\varepsilon} K(z, 0)\left(K(z, 0)^{*}-K(0,0)^{*}\right) z^{-1} d z .
\end{aligned}
$$

Lemma 2.1, (4) with $k=1$, and (5) now give $\left|H_{n n}\right| \leqq 800 R^{4} r^{-5} \varepsilon$. If $m>n$, then:

$$
H_{m n}=\frac{\varepsilon^{n-m}}{2 \pi i K(0,0)^{2}} \int_{|z|=\varepsilon} K(z, 0) K(z, 0)^{*} z^{m-n-1} d z .
$$

As before, we may replace the second occurrence of $K(z, 0)$ in the integral by $K(z, 0)$ minus its Taylor expansion, this time as far as the term in $z^{m-n-1}$. Then by (5), Lemma 2.1, and (4) with $k=m-n$ :

$$
\begin{aligned}
\left|H_{m n}\right| & \leqq \varepsilon^{m-n} 1.01 \frac{16 \pi R^{2} \gamma}{r^{2}} \frac{7 R^{2}(m-n+1)}{r(r-2 \varepsilon)^{m-n+1} \gamma} \\
& \leqq 400 R^{4} r^{-5} \varepsilon(|m-n|+1)(1 / 99998)^{|m-n|-1}
\end{aligned}
$$

since $\varepsilon<10^{-5} r$. This holds similarly for $m<n$. Combining the cases $m=n, m>n$, and $m<n$, we see that:

$$
\begin{aligned}
\|H\| & \leqq \frac{\varepsilon R^{4}}{r^{5}}\left(800+2 \times 400\left(2+\frac{3}{99998}+\frac{4}{(99998)^{2}}+\cdots\right)\right) \\
& \leqq 2401 R^{4} r^{-5} \varepsilon \leqq 2401(R / r)^{5} \gamma^{-1} \varepsilon .
\end{aligned}
$$


So $\left[\begin{array}{cc}D & E^{H} \\ E & F\end{array}\right]$ has norm at most $\|G\|+\|H\| \leqq 2500(R / r)^{5} \gamma^{-1} \varepsilon$. Hence each of $\|\vec{D}\|,\|E\|,\|F\| \leqq 2500(R / r)^{5} \gamma^{-1} \varepsilon \leqq 1 / 40$.

To summarise: we have shown that:

$$
\begin{aligned}
& \|A\|,\|B\|^{2},\|C\|,\|D\|,\|E\|,\|F\| \leqq 2500(R / r)^{5} \gamma^{-1} \varepsilon ; \\
& \|A\|,\|B\|,\|C\|,\|D\|,\|E\|,\|F\| \leqq 1 / 40 .
\end{aligned}
$$

In particular we have verified that $M$ is a bounded matrix: indeed that $\|M\| \leqq 3 / 40<1$. Thus $T=I+M$ is invertible, and Proposition 1.5 applies.

Our next step is to calculate the top left-hand block of the inverse of $T$. Since $T^{-1}=I-M+M^{2}-M^{3}+\cdots$, this top left-hand block is:

$$
\begin{aligned}
S= & I \\
& -A \\
& +A^{2}+B^{H} B+C^{H} C \\
& -A^{3}-A B^{H} B-A C^{H} C-B^{H} B A-B^{H} D B-B^{H} E^{H} C-C^{H} C A \\
& -C^{H} E B-C^{H} F C \\
& +\cdots .
\end{aligned}
$$

The row of this expression containing products of degree $n(n \geqq 4)$ consists of $3^{n-1}$ terms. Each of these terms has norm at most $(2500)^{2}(R / r)^{10} \gamma^{-2} \varepsilon^{2}(1 / 40)^{n-4}$ by (10). Hence $S=I-A+B^{H} B$ with error:

$$
\begin{aligned}
& \frac{(2500)^{2} \varepsilon^{2} R^{10}}{\gamma^{2} r^{10}}\left(1+1+\frac{1}{40}+1+\frac{1}{40}+1+1+\frac{1}{40}+\frac{1}{40}+\frac{1}{40}+\frac{1}{40}\right. \\
& \left.+27\left(1+\frac{3}{40}+\left(\frac{3}{40}\right)^{2}+\cdots\right)\right) \leqq 3.10^{8}(R / r)^{10} \gamma^{-2} \varepsilon^{2} .
\end{aligned}
$$

Using (7) and (9), we have:

$$
S=\left[\delta_{m n}-2 \pi \varepsilon u_{m}(0)^{*} u_{n}(0)+\frac{\varepsilon}{2 \pi K(0,0)^{2}} \int \frac{K(z, 0) u_{m}^{*} d s}{z} \int \frac{K(z, 0)^{*} u_{n} d s}{z^{*}}\right]
$$

with error $200 R^{2} r^{-3} \gamma^{-1} \varepsilon^{2}+20000 R^{6} r^{-8} \varepsilon^{2}+3.10^{8}(R / r)^{10} \gamma^{-2} \varepsilon^{2} \leqq 4.10^{8}(R / r)^{10} \gamma^{-2} \varepsilon^{2}$. Here and subsequently all integrals are taken round $\partial \Omega$.

Finally we apply Proposition 1.5 , which says that $1 /\left(2 \pi \gamma_{1}\right)=$ $\sum S_{m n} u_{m}(\infty) u_{n}(\infty)^{*}\left(\right.$ since $v_{n}(\infty)=0$ for all $\left.n\right)$. Hence:

$$
\begin{gathered}
\frac{1}{2 \pi \gamma_{1}}=\sum\left|u_{n}(\infty)\right|^{2}-2 \pi \varepsilon\left|\sum u_{n}(0)^{*} u_{n}(\infty)\right|^{2} \\
+\frac{\varepsilon}{2 \pi K(0,0)^{2}}\left|\sum\left(u_{n}(\infty) \int \frac{K(z, 0) u_{n}^{*} d s}{z}\right)\right|^{2}
\end{gathered}
$$


with error $4.10^{8}(R / r)^{10} \gamma^{-2} \varepsilon^{2} \sum\left|u_{m}(\infty)\right|^{2}=4.10^{8}(R / r)^{10} \gamma^{-2} \varepsilon^{2} /(2 \pi \gamma)$. Multiplying by $2 \pi \gamma$ and using the fact that $\sum u_{n}(z) u_{n}(\zeta)^{*}=K(z, \zeta)$, we have:

$$
\begin{aligned}
& \frac{\gamma}{\gamma_{1}}=1-4 \pi^{2} \gamma \varepsilon|K(0, \infty)|^{2}+\frac{\gamma \varepsilon}{K(0,0)^{2}}\left|\int \frac{K(z, 0) K(z, \infty)^{*} d s}{z}\right|^{2} \\
& \quad \text { with error } 4.10^{8}(R / r)^{10} \gamma^{-2} \varepsilon^{2} .
\end{aligned}
$$

Now the last term simplifies. On $\partial \Omega, f(z) \psi(z) d z \geqq 0$, so that $d s=$ $(\psi(z) /|\psi(z)|) f(z) d z=\left(K(z, \infty) / K(z, \infty)^{*}\right)(f(z) / i) d z$. Therefore:

$$
\begin{aligned}
& \int \frac{K(z, 0) K(z, \infty)^{*} d s}{z}=\frac{1}{i} \int \frac{K(z, 0) K(z, \infty) f(z) d z}{z} \\
& \quad=-2 \pi K(0,0) K(0, \infty) f(0)
\end{aligned}
$$

since $K(z, 0) K(z, \infty) f(z)$ is analytic on $\bar{\Omega}$ and vanishes at $\infty$. Substituting in (11), we have:

$$
\frac{\gamma}{\gamma_{1}}=1-4 \pi^{2} \gamma \varepsilon|K(0, \infty)|^{2}\left\{1-|f(0)|^{2}\right\} \text { with error } 4.10^{8}(R / r)^{10} \gamma^{-2} \varepsilon^{2} .
$$

Now $4 \pi^{2} \gamma \varepsilon|K(0, \infty)|^{2}\left\{1-|f(0)|^{2}\right\} \leqq 4 \pi^{2} \gamma \varepsilon K(0,0) K(\infty, \infty) \leqq 8(R / r)^{2} \gamma^{-1} \varepsilon<$ $10^{-4}$. Also $4.10^{8}(R / r)^{10} \gamma^{-2} \varepsilon^{2} \leqq 1 / 25$. So we can invert to obtain:

$$
\begin{aligned}
& \frac{\gamma_{1}}{\gamma}=1+4 \pi^{2} \gamma \varepsilon|K(0, \infty)|^{2}\left\{1-|f(0)|^{2}\right\} \text { with error } 10^{9}(R / r)^{10} \gamma^{-2} \varepsilon^{2} ; \\
& \gamma_{1}=\gamma+4 \pi^{2} \gamma^{2} \varepsilon|K(0, \infty)|^{2}\left\{1-|f(0)|^{2}\right\} \\
& =\gamma+2 \pi \varepsilon|\psi(0)|\left\{1-|f(0)|^{2}\right\} \text { with error } 10^{9}(R / r)^{10} \gamma^{-1} \varepsilon^{2} .
\end{aligned}
$$

It is as well to explain the curious choice of the functions $v_{n}$ in the above proof. The only essential property of $v_{n}$ we used is that it vanishes at $\infty$ and is analytic on $\bar{\Omega}$ except for a pole at 0 near which $v_{n}(z)=(2 \pi)^{-1 / 2} \varepsilon^{n-1 / 2} z^{-n}+\cdots$. The simpler choice $v_{n}(z)=$ $(2 \pi)^{-1 / 2} \varepsilon^{n-1 / 2} z^{-n}$ shortens the proof but yields an error bound dependent on the length of $\partial \Omega$, which would have been unsuitable for the next section.

3. Extension to arbitrary compact sets. We shall now show how the above results extend to arbitrary compact sets $E$. In particular, we show how to define the Garabedian function of $E$, thus solving a problem considered in [2] and [3].

Let $E$ be compact. We shall suppose meantime that $\gamma(E)>0$. $E$ can be expressed as the intersection of a decreasing sequence $\left\{E_{n}\right\}$ in $\mathscr{S}$. Hence $\psi_{E_{n}}$ and $a_{E_{n}}$ are defined. Fix $\zeta \in \Omega(E)$, and choose $n_{0}$ so that $\zeta \in \Omega\left(E_{n}\right)$ whenever $n>n_{0}$. By Theorem 2.2 there exist $\varepsilon_{0}>$ $0, k>0$, such that $\forall n>n_{0}, \forall \varepsilon<\varepsilon_{0}$ :

$$
\left|\gamma\left(E_{n} \cup D(\zeta ; \varepsilon)\right)-\gamma\left(E_{n}\right)-\varepsilon a_{E_{n}}(\zeta)\right| \leqq k \varepsilon^{2} .
$$


That is, for all $\varepsilon<\varepsilon_{0}$, the sequence $\left\{\varepsilon \alpha_{E_{n}}(\zeta)\right\}_{n>n_{0}}$, considered as an element of the Banach space of bounded sequences with the supremum norm, is within a distance $k \varepsilon^{2}$ of the sequence $\left\{\gamma\left(E_{n} \cup D(\zeta ; \varepsilon)\right)-\right.$ $\left.\gamma\left(E_{n}\right)\right\}_{n>n_{0}}$, which converges to $\gamma(E \cup D(\zeta ; \varepsilon))-\gamma(E)$ by 1.1. Thus $\left\{a_{E_{n}}(\zeta)\right\}$ is within a distance $k \varepsilon$ of the closed subspace $c$ of convergent sequences, for all $\varepsilon$, and is therefore itself in $c$. Call its limit $a_{E}(\zeta)$. $a_{E}$ is the slope function of $E$. Letting $n \rightarrow \infty$ in (12) now gives, for all $\varepsilon<\varepsilon_{0}$ :

$$
\left|\gamma(E \cup D(\zeta ; \varepsilon))-\gamma(E)-\varepsilon a_{E}(\zeta)\right| \leqq k \varepsilon^{2} .
$$

This shows also that the limit $a_{E}(\zeta)$ is independent of the choice of the sequence $\left\{E_{n}\right\}$.

Now, for each $n,\left|\psi_{E_{n}}(\zeta)\right|=a_{E_{n}}(\zeta) /\left(2 \pi\left\{1-\left|f_{E_{n}}(\zeta)\right|^{2}\right\}\right)$, and this converges pointwise in $\Omega(E)$. Moreover, $\left\{\psi_{E_{n}}\right\}$ is a normal sequence, since, if $F$ is a compact subset of $\Omega(E),\left\{\psi_{E_{n}}\right\}$ is uniformly bounded on $F$ by the remark following Lemma 2.1. It follows that for some sequence $\lambda_{n}$ of points on the unit circle, $\left\{\lambda_{n} \psi_{E_{n}}\right\}$ converges uniformly on compact subsets of $\Omega(E)$. In fact we may take $\lambda_{n}=1$, since $\psi_{E_{n}}(\infty)=1 /(2 \pi i)$. So $\left\{\psi_{E_{n}}\right\}$ converges uniformly on compact sets. Call its limit, $\left\{\psi_{E}\right\}$, the Garabedian function of $E$. Hence also $a_{E_{n}}(\zeta)=$ $2 \pi\left|\psi_{E_{n}}(\zeta)\right|\left\{1-\left|f_{E_{n}}(\zeta)\right|^{2}\right\}$ converges uniformly on compact sets (and not merely pointwise, as ascertained already).

Now suppose that $\gamma(E)=0$. We define $\psi_{E}(\zeta)=1 /(2 \pi i), a_{E}(\zeta)=1$ for $\zeta \in \Omega(E)$. This is consistent with the relation $a_{E}(\zeta)=2 \pi\left|\psi_{E}(\zeta)\right|$ $\left\{1-\left|f_{E}(\zeta)\right|^{2}\right\}$ since $f_{E}(\zeta)=0 . \quad \gamma(E \cup D(\zeta ; \varepsilon))=\varepsilon$ for all $\varepsilon>0$ by 1.3 , and so the relation $\gamma(E \cup D(\zeta ; \varepsilon))=\gamma(E)+\varepsilon a_{E}(\zeta)+O\left(\varepsilon^{2}\right)$ holds trivially. If $\left\{E_{n}\right\}$ is a sequence in $\mathscr{S}$ decreasing to $E$, then $\psi_{E_{n}}(\zeta) \rightarrow$ $1 /(2 \pi i)=\psi_{E}(\zeta)$ uniformly on compact sets by the remark following Lemma 2.1.

Finally, if $E$ is compact, and $\left\{E_{n}\right\}$ is any sequence of compact sets decreasing to $E$, the same working as above shows that $\psi_{E_{n}} \rightarrow$ $\psi_{E}$ and $a_{E_{n}} \rightarrow a_{E}$ uniformly on compact sets.

We have therefore proved:

THEOREM 3.1. The Garabedian function $\psi_{E}(\zeta)$ and the slope function $a_{E}(\zeta)$ can be defined for all compact sets $E$, in such a way that:

(1) The definitions coincide with the existing meanings if $E \in \mathscr{S}$

(2) If $\left\{E_{n}\right\}$ is a sequence of compact sets decreasing to $E$, then $\psi_{E_{n}} \rightarrow \psi_{E}$ and $a_{E_{n}} \rightarrow a_{E}$ uniformly on compact subsets of $\Omega(E)$;

(3) $\gamma(E \cup D(\zeta ; \varepsilon))=\gamma(E)+\varepsilon a_{E}(\zeta)+O\left(\varepsilon^{2}\right)$ for all $\zeta \in \Omega(E)$, and the bound in the error term depends only on $\gamma(E)$ and on the ratio of the greatest and least distances of points of $E$ from $\zeta$; and

(4) $a_{E}(\zeta)=2 \pi|\psi(\zeta)|\left\{1-|f(\zeta)|^{2}\right\}$ for all $\zeta \in \Omega(E)$. 
The slope function is related to the problem of subadditivity of $\gamma$. If $E$ is connected, then $a_{E}(\zeta) \leqq 1$ : this is a re-statement of Bieberbach's distortion theorem. Subadditivity of $\gamma$ would obviously imply $a_{E}(\zeta) \leqq$ 1 for all compact $E$.

I should like to thank Dr. A. M. Davie for his invaluable supervision.

Added in proof. N. Suita recently has independently proved the uniqueness of the Garabedian function much more simply ("On a metric induced by Analytic Capacity," Kōdai Math. Sem. Rep. 25 (1973), 215-218).

\section{REFERENCES}

1. T. W. Gamelin, Uniform Algebras, Prentice-Hall, Englewood Cliffs, N.J., 1969.

2. J. Garnett, Analytic capacity and measure, Springer Lecture Notes in Mathematics, 297 (1972).

3. S. Y. Havinson, Analytic capacity of sets, joint nontriviality of various classes of analytic functions, and the Schwarz lemma in arbitrary domains, Mat. Sb. (N.S.), 54 (96) (1961), 3-50; also, Amer. Math. Soc. Translations, ser. 2, 43 (1964), 215-266.

Received December 26, 1972. This paper contains material which will appear in the author's doctoral thesis, supervised by Dr. A. M. Davie at the University of Edinburgh. The work is supported by a scholarship given by the Carnegie Trust for the Universities of Scotland. 


\section{PACIFIC JOURNAL OF MATHEMATICS}

\section{EDITORS}

RICHARD ARENS (Managing Editor)

University of California

Los Angeles, California 90024

R. A. BeAumont

University of Washington

Seattle, Washington 98105
J. DugundJI*

Department of Mathematics

University of Southern California

Los Angeles, California 90007

D. Gilbarg and J. Milgram

Stanford University

Stanford, California 94305

\section{ASSOCIATE EDITORS}

E. F. BECKENBACH

B. H. NEUMANN

F. WOLF

K. YoSHIDA

\section{SUPPORTING INSTITUTIONS}

UNIVERSITY OF BRITISH COLUMBIA
CALIFORNIA INSTITUTE OF TECHNOLOGY
UNIVERSITY OF CALIFORNIA
MONTANA STATE UNIVERSITY
UNIVERSITY OF NEVADA
NEW MEXICO STATE UNIVERSITY
OREGON STATE UNIVERSITY
UNIVERSITY OF OREGON
OSAKA UNIVERSITY

UNIVERSITY OF BRITISH COLUMBIA CALIFORNIA INSTITUTE OF TECHNOLOGY UNIVERSITY OF CALIFORNIA MONTANA STATE UNIVERSITY NEW MEXICO STATE UNIVERSITY UNIVERSITY OF OREGON OSAKA UNIVERSITY
UNIVERSITY OF SOUTHERN CALIFORNIA STANFORD UNIVERSITY UNIVERSITY OF TOKYO UNIVERSITY OF UTAH WASHINGTON STATE UNIVERSITY UNIVERSITY OF WASHINGTON AMERICAN MATHEMATICAL SOCIETY NAVAL WEAPONS CENTER

* C. R. DePrima California Institute of Technology, Pasadena, CA 91109, will replace J. Dugundji until August 1974. 


\section{Pacific Journal of Mathematics}

\section{Vol. 51, No. $1 \quad$ November, 1974}

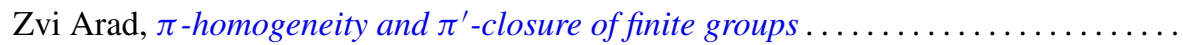

Ivan Baggs, A connected Hausdorff space which is not contained in a maximal

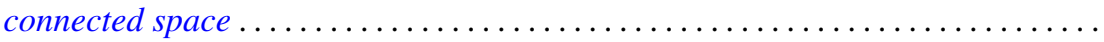

Eric Bedford, The Dirichlet problem for some overdetermined systems on the unit ball in $C^{n}$

R. H. Bing, Woodrow Wilson Bledsoe and R. Daniel Mauldin, Sets generated by

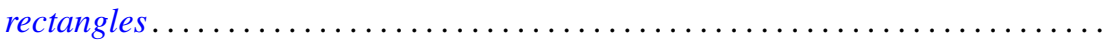

Carlo Cecchini and Alessandro Figà-Talamanca, Projections of uniqueness for

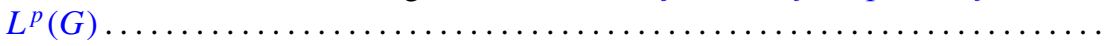

Gokulananda Das and Ram N. Mohapatra, The non absolute Nörlund summability of Fourier series .

Frank Rimi DeMeyer, On separable polynomials over a commutative ring ........ Richard Detmer, Sets which are tame in arcs in $E^{3} \ldots \ldots \ldots \ldots \ldots \ldots \ldots \ldots$

William Erb Dietrich, Ideals in convolution algebras on Abelian groups ..........

Bryce L. Elkins, A Galois theory for linear topological rings .................

William Alan Feldman, A characterization of the topology of compact convergence on $C(X)$.

Hillel Halkin Gershenson, A problem in compact Lie groups and framed cobordism

Samuel R. Gordon, Associators in simple algebras.

Marvin J. Greenberg, Strictly local solutions of Diophantine equations

Jon Craig Helton, Product integrals and inverses in normed rings . . . . . . . . . . . .

Domingo Antonio Herrero, Inner functions under uniform topology . . .

Jerry Alan Johnson, Lipschitz spaces .

Marvin Stanford Keener, Oscillatory solutions and multi-point boundary value

functions for certain nth-order linear ordinary differential equations.

John Cronan Kieffer, A simple proof of the Moy-Perez generalization of the

Shannon-McMillan theorem .......................

Joong Ho Kim, Power invariant rings

Gangaram S. Ladde and V. Lakshmikantham, On flow-invariant sets .

Roger T. Lewis, Oscillation and nonoscillation criteria for some self-adjoint even

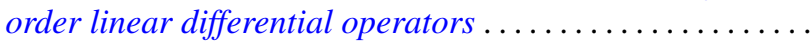

Jürg Thomas Marti, On the existence of support points of solid convex sets ..

John Rowlay Martin, Determining knot types from diagrams of knots . .

James Jerome Metzger, Local ideals in a topological algebra of entire functions

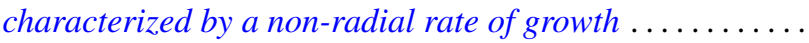

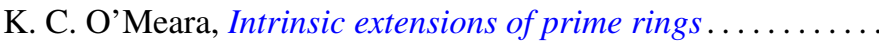

Stanley Poreda, A note on the continuity of best polynomial approximations ..

Robert John Sacker, Asymptotic approach to periodic orbits and local prolongations

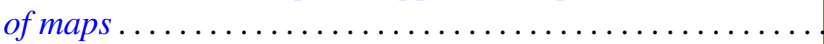

Eric Peter Smith, The Garabedian function of an arbitrary compact set . .

Arne Stray, Pointwise bounded approximation by functions satisfying a side condition

John St. Clair Werth, Jr., Maximal pure subgroups of torsion complete abelian

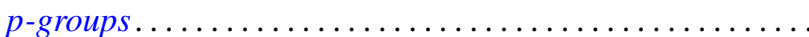

